\title{
Self-Sustained Cascading Coalescence in Surface Condensation
}

\section{Journal Article}

\section{Author(s):}

Sharma, Chander Shekhar; Lam, Cheuk W.E.; Milionis, Athanasios; Eghlidi, Hadi; Poulikakos, Dimos

Publication date:

2019-07-31

Permanent link:

https://doi.org/10.3929/ethz-b-000360087

\section{Rights / license:}

In Copyright - Non-Commercial Use Permitted

\section{Originally published in:}

ACS Applied Materials \& Interfaces 11(30), https://doi.org/10.1021/acsami.9b07673 


\title{
Self-Sustained Cascading Coalescence in Surface Condensation
}

\author{
Chander Shekhar Sharma, *\$॰ Cheuk Wing Edmond Lam, Athanasios Milionis, Hadi Eghlidi, \\ and Dimos Poulikakos*(1) \\ Laboratory of Thermodynamics in Emerging Technologies, Department of Mechanical and Process Engineering, ETH Zurich, 8092 \\ Zurich, Switzerland
}

\section{Supporting Information}

ABSTRACT: Sustained dropwise condensation of water requires rapid shedding of condensed droplets from the surface. Here, we elucidate a microfluidic mechanism that spontaneously sweeps condensed microscale droplets without the need for the traditional droplet removal pathways such as use of superhydrophobicity for droplet rolling and jumping and utilization of wettability gradients for directional droplet transport among others. The mechanism involves self-generated, directional, cascading coalescence sequences of condensed microscale droplets along standard hydrophobic microgrooves. Each sequence appears like a spontaneous zipping process, can sweep droplets along the microgroove at speeds of up to $\sim 1 \mathrm{~m} / \mathrm{s}$, and can extend for lengths more than 100 times the microgroove width. We investigate this phenomenon through high-speed in situ microscale condensation observations and demonstrate that it is enabled by rapid oscillations of a condensate meniscus formed locally in a filled microgroove and pinned on its edges. Such oscillations are in turn spontaneously

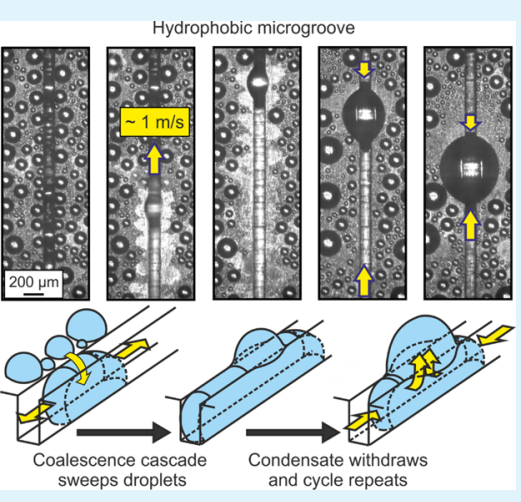
initiated by coalescence of an individual droplet growing on the ridge with the microgroove meniscus. We quantify the coalescence cascades by characterizing the size distribution of the swept droplets and propose a simple analytical model to explain the results. We also demonstrate that, as condensation proceeds on the hydrophobic microgrooved surface, the coalescence cascades recur spontaneously through repetitive dewetting of the microgrooves. Lastly, we identify surface design rules for consistent realization of the cascades. The hydrophobic microgrooved textures required for the activation of this mechanism can be realized through conventional, scalable surface fabrication methods on a broad range of materials (we demonstrate with aluminum and silicon), thus promising direct application in a host of phasechange processes.

KEYWORDS: condensation, hydrophobic, coalescence, cascade, microgrooves

\section{INTRODUCTION}

Electricity, ${ }^{1}$ potable drinking water, ${ }^{2}$ and information technology $^{3}$ count as among the critical demands of modern human society. The diverse industrial activities that endeavor to meet these needs, thermal power generation, ${ }^{4}$ water desalination, ${ }^{5}$ water harvesting, ${ }^{6,7}$ and thermal management of electronic devices $^{8}$ among others, involve heterogeneous condensation of water as a critical physical process. It is well known that hydrophobic and superhydrophobic surfaces cause water to condense as distinct droplets that are periodically shed to achieve significant improvement in the thermodynamic efficiency of this phase-change process compared to hydrophilic surfaces wherein condensed water forms a continuous liquid film. ${ }^{9}$ Inspired by natural hydrophobic surfaces capable of passive removal of droplets, multiple artificial surfaces have been proposed, which achieve droplet shedding through gravity by using hydrophobic substrates ${ }^{9,10}$ or by causing extreme sliding through lubricant-infused texturing. ${ }^{11-14}$ Further enhancement in dropwise condensation has been achieved through gravity-independent droplet removal by utilizing droplet coalescence-induced jumping on super- hydrophobic surfaces ${ }^{15-18}$ or by causing directional droplet movement with capillarity gradients. ${ }^{19,20}$

In this work, we investigate a mechanism that spontaneously sweeps condensate droplets from hydrophobic surfaces without the need for the traditional droplet removal pathways mentioned above. It involves a self-generated cascading sequence of droplet coalescences along standard hydrophobic microgrooves, which can proceed at a speed of up to $\sim 1 \mathrm{~m} / \mathrm{s}$ and can sweep droplets over large ridge areas between microgrooves. We explain the underlying physics for these coalescence cascades through careful high-speed in situ observations of condensation at a microscale and identify design rules for their consistent realization. Such droplet sweeps present an alternative approach for gravity-independent refreshment of the condensing surface while requiring only facile and scalable surface fabrication on a wide range of materials, including metals, thus avoiding a more elaborate

Received: May 2, 2019

Accepted: July 4, 2019

Published: July 4, 2019 
surface preparation necessary in many of the traditional approaches for passive droplet removal from the surface. ${ }^{17,21}$

\section{RESULTS AND DISCUSSION}

2.1. Hydrophobic Microgrooved Surface. We investigate the condensation of water on hydrophobic microgrooved surfaces as shown in Figure 1. We fabricate such microgrooves
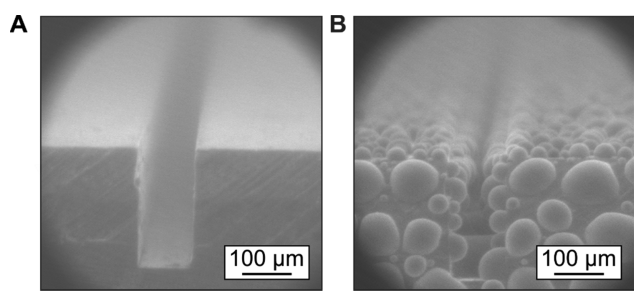

Figure 1. (A) Microgroove machined in aluminum and coated with polytetrafluoroethylene (PTFE). (B) Hydrophobicity of the microgroove toward condensed microdroplets as observed in ESEM. The vapor pressure was set at $0.87 \mathrm{kPa}$, and the cooling stage was set at 2 ${ }^{\circ} \mathrm{C}$. (See the Supporting Information for details on ESEM observations.)

primarily on a metal (aluminum) due to the obvious importance of metals in energy applications. Figure 1A shows the morphology of such a microgroove as obtained through conventional milling in aluminum followed by adding a hydrophobic coating. Similar microgrooves can also be obtained from silicon through standard etching methods (see the Experimental Section and Section S1). Figure 1B shows the hydrophobicity of the surface at a microscale as observed through in situ condensation observation using ESEM.

2.2. Coalescence Cascade. The mechanism manifests itself as a self-propagating, cascading sequence of droplet coalescence events advancing in a zipping manner during condensation of water on a standard hydrophobic microgrooved surface, as shown in Figure 2A. Condensation on such microgrooved surfaces proceeds through spatially uniform nucleation and growth of condensate droplets within and outside the microgrooves. The nucleation and growth of droplets inside and outside the microgrooves is likely to be similar due to similarity in curvature, chemistry, and roughness of surfaces inside and outside the microgrooves. ${ }^{17,22}$ Eventually, when a microgroove is filled with the condensate at any location, a droplet growing outside the microgroove on the ridge may coalesce with the condensate in the microgroove (Figure 2, panels i and ii). Unexpectedly, however, instead of such coalescence causing the removal of only this individual droplet from the ridge, we observe that such an individual coalescence event can trigger a rapid sequence of similar coalescence events along the length of the microgroove. ${ }^{23}$ High-speed imaging reveals a cascading coalescence sequence that proceeds at speeds of up to $1 \mathrm{~m} / \mathrm{s}$ while sweeping condensate droplets from a large area along the ridge in a zipping-like motion (see panels ii-ix in Figure 2A). As indicated by the gravity vector in the first panel, this effect can also proceed against gravity. The sequence eventually culminates with the microgroove filled with the condensate collected from the coalescing droplets and any excess condensate located in multiple bulges along the filled length of the microgroove (panels ix and $\mathrm{x}$ in Figure 2A). The small bulges eventually collapse into the microgroove (panel $\mathrm{xi}$ in Figure $2 \mathrm{~A}$ ) collecting the excess condensate in a single large bulge (not visible in the figure) at the completion of the coalescence sequence.

The propagation of this zipping-like coalescence sequence is achieved by the oscillations of the condensate meniscus inside the microgroove, as elucidated through high-speed snapshots in Figure $2 \mathrm{~B}$ and corresponding schematics shown in Figure $2 \mathrm{C}$. We observe that these oscillations are always initiated from

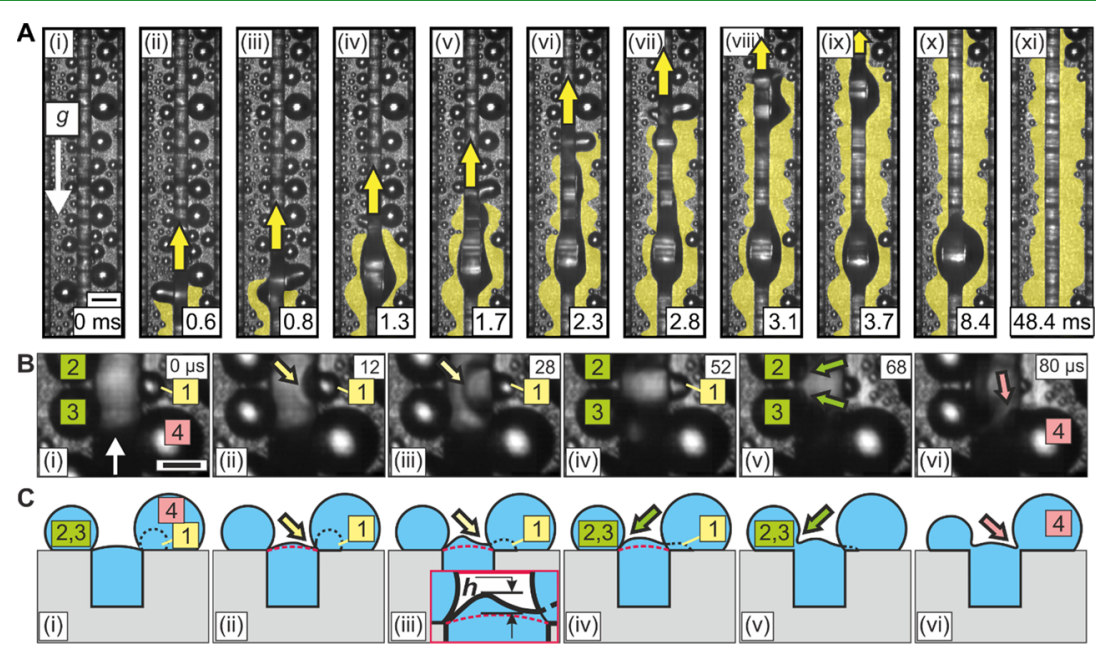

Figure 2. Microdroplet coalescence cascade. (A) High-speed microdroplet coalescence sequence along a $\sim 100 \mu \mathrm{m}$ wide and $\sim 200 \mu \mathrm{m}$ deep hydrophobic microgroove. Substrate: aluminum; coating: polytetrafluoroethylene (PTFE). The yellow color indicates the coalescence cascade progress. $g$ indicates gravity. The scale bar represents $200 \mu \mathrm{m}$. Images are captured at 10,000 fps (frames per second) (Movie S1). (B) High-speed snapshots captured at 250,000 fps demonstrating the spontaneous initiation and sustenance of the coalescence sequence. The scale bar represents $100 \mu \mathrm{m}$. The white arrow indicates the viewing direction for (C). (See the Experimental Section and Section S2 for details of the experimental setup and procedure.) (C) Schematics corresponding to images in (B). Droplet 1 spontaneously coalesces with the condensate meniscus pinned at microgroove edges and initiates the coalescence sequence [see yellow arrows in panel ii in (B) and (C)]. As this coalescence proceeds, the meniscus rises [by amplitude as shown in the inset figure in panel iii of $(\mathrm{C})$ ]. This appears as a capillary ripple traveling along the microgroove meniscus [panels ii-iv in (B)]. The oscillating meniscus first catches droplets 2 and 3 [green arrows in panel $\mathrm{v}$ in (B) and (C)], which in turn triggers the coalescence of droplet 4 [pink arrows in panel vi in (B) and (C)] thus propagating the coalescence sequence. (Movie S2.) 

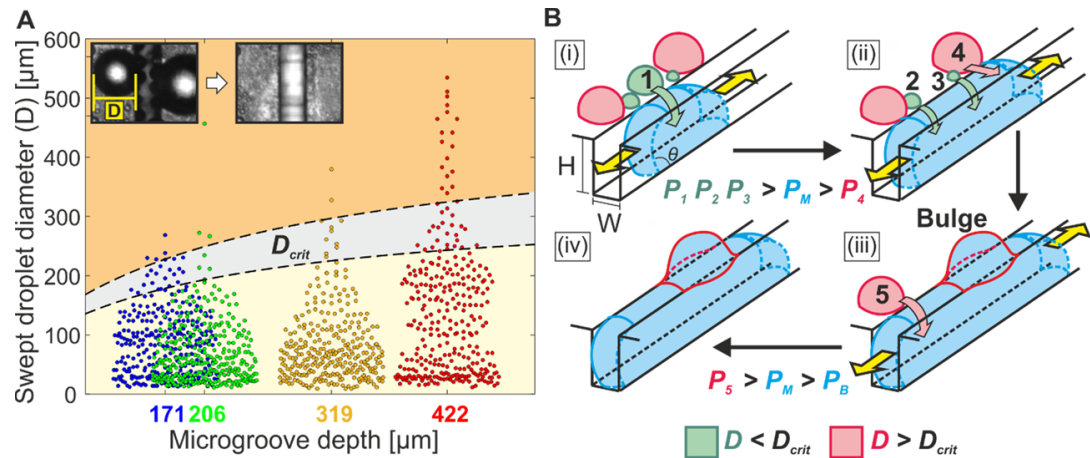

Figure 3. (A) Beeswarm distribution plots of swept droplet diameters ( $D$, defined in inset) for microgrooves of widths of $\sim 100 \mu \mathrm{m}$ and depths of $\sim 171$ to $422 \mu \mathrm{m}$ machined in aluminum and coated with PTFE. The grey zone demarcates the limit estimate $D_{\text {crit }}$. Each beeswarm plot is based on $\sim 300$ measurements and is shown in different colors for clarity. In the beeswarm distribution, the horizontal spread at any ordinate $D$ indicates the relative proportion of droplets of diameter $D$ swept by the cascading coalescence sequence. The yellow zone below the $D_{\text {crit }}$ limit represents the range of droplet sizes swept by the coalescence sequence progressing in a partially filled microgroove. The orange zone above $D_{\text {crit }}$ indicates droplet sizes swept by the coalescence sequence proceeding in a completely filled microgroove or in the presence of a large bulge. (B) Schematic illustrating that droplets with $D<D_{\text {crit }}$ (droplets 1,2 , and 3 in green) are readily absorbed by the advancing meniscus [panels (i) and (ii)] due to a favorable Laplace pressure difference with the microgroove meniscus $\left(P_{1}, P_{2}, P_{3}>P_{\mathrm{M}}\right)$. A droplet with $D>D_{\text {crit }}$ (droplet 4 in red with $\left.P_{4}<P_{\mathrm{M}}\right)$ forms a bulge. The bulge causes the sweeping of subsequent droplets with $D>D_{\text {crit }}$ [e.g., droplet 5 in panels (iii) and (iv)]. $P_{\mathrm{B}}$ represents Laplace pressures inside the bulge. The legend at the bottom defines the color scheme for droplets.

a completely filled part of microgroove wherein a meniscus pinned at the edges of the microgroove exists. When a droplet growing in the ridge area and near the microgroove edge, for instance droplet 1 in Figure 2B, coalesces with the pinned meniscus, this otherwise stable meniscus ${ }^{24}$ gets perturbed. The droplet has higher Laplace pressure as compared to the condensate within the microgroove due to the much higher curvature of the droplet. This causes the droplet to drain into the condensate within the microgroove, ${ }^{10}$ and the associated fluid inertia drives the meniscus beyond its equilibrium position. $^{25}$ The resulting competition between fluid inertia and surface tension results in a perturbation with an amplitude of the order of $\sim 10 \mu \mathrm{m}$ (defined by $h$ in Figure 2, panel iii; see Section S3 for details) that rapidly travels as a capillary ripple across the width of the microgroove. ${ }^{26}$ The phase velocity $u$ of the capillary-gravity waves created at any interface is given by $u^{2}=\sigma k / \rho+g / k$ where $\sigma$ and $\rho$ are the surface tension and density of water, respectively, and $k=2 \pi / \lambda$ is the wavenumber with $\lambda$ as the wavelength of the capillary ripple. ${ }^{26,27}$ However, surface tension dominates at small length scales relevant here, and thus the meniscus perturbation is a pure capillary ripple traveling across the pinned meniscus at a phase velocity of $u=\sqrt{2 \pi \sigma / \lambda \rho}$. While the coalescence continues, the capillary ripple reflects from the pinned edge of the meniscus and triggers transverse oscillations similar to those of a sessile droplet with a pinned contact line..$^{25,28,29}$ Due to the small length scales involved, these meniscus oscillations proceed with a short characteristic time period $\tau_{\mathrm{c}}=\sqrt{\rho \lambda^{3} / 2 \pi \sigma} \approx 10 \mu \mathrm{s}$ thus necessitating high-speed imaging to capture them (Section S4). This time period reflects the fact that these meniscus oscillations are governed by a competition between fluid inertial and capillary forces, similar to the time scale of liquid droplet coalescence. ${ }^{30,31}$ These meniscus oscillations persist due to the relatively long relaxation time inherent in sessile droplet coalescence ${ }^{32-34}$ and catch further droplets along the edge of the microgroove, as shown in Figure 2B,C, even before the original droplet coalescence event is completed. This is evident from the initiation of coalescence of droplets 2 and 3, much before the coalescence of droplet 1 is completed. This process repeats, and the coalescence sequence propagates along the microgroove. We observed that the overall speed of this propagation depends upon the amount of condensate present in the microgroove immediately prior to the initiation of the coalescence sequence. A microgroove that is fully filled with condensate causes the sequence to proceed at speeds as high as $1 \mathrm{~m} / \mathrm{s}$. The propagation speed is lower if the microgroove is only partially filled with the condensate because, in this case, the microgroove needs to be progressively filled by advancing the meniscus within the microgroove in order to catch the downstream droplets on the ridge. (See Section S5 for further details.)

Irrespective of the state of the microgroove before the initiation of the cascading coalescence sequence, once initiated, the sequence propagates as long as droplets are available within and outside the microgroove to feed the meniscus advancing within the microgroove. This results in coalescence sequences that can propagate for lengths more than 100 times the microgroove width. Any coalescence event between the advancing meniscus and an individual droplet induces oscillations that are essentially local. These oscillations need to de-pin the meniscus within the microgroove and advance it sufficiently to catch the next downstream droplet. In the absence of such droplets, the oscillations are exponentially attenuated by the bulk viscous dissipation within the liquid ${ }^{10}$ with a characteristic time of $\tau_{\mathrm{r}}=\eta \lambda^{3} / \sigma W^{3}$, which is of the order of $\sim 0.01 \mu \mathrm{s} \ll \tau_{\mathrm{c}}$ (Section S6). Here, $\eta$ is the fluid viscosity, and $W$ is the microgroove width. However, the resulting termination of the coalescence sequence is only temporary, and the phenomenon self-initiates again when droplets become available through continuous condensation.

2.3. Modeling and Swept-Droplet Distribution. The coalescence sequence sweeps droplets of a range of sizes from the ridge area as it propagates along the microgroove. We investigate this aspect for varying microgroove geometries by analyzing the size distribution of droplets swept during the coalescence cascades. Figure 3A shows such distributions for four microgrooves with the same widths but increasing depths from 171 to $422 \mu \mathrm{m}$. The swept-droplet size is defined by droplet diameter $D$ as shown in inset of Figure 3A. The 

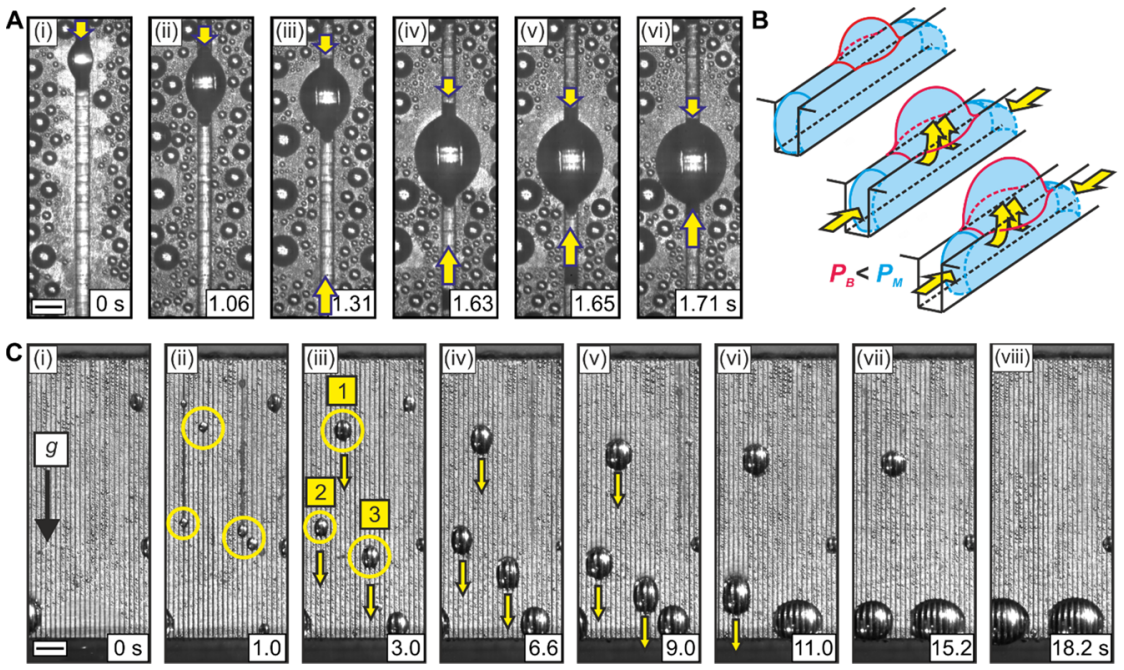

Figure 4. Periodicity of the zipping-like cascading coalescence sequence: (A) a single bulge and filled microgroove are formed after the conclusion of the coalescence sequence (panel i). Subsequently, the bulge induces dewetting of the microgroove through condensate withdrawal as indicated by yellow arrows. Scale bar: $200 \mu \mathrm{m}$. Images are captured at $1000 \mathrm{fps}$. (B) Schematic showing the condensate withdrawal process along with an overall pressure difference driving this process. (C) Zoomed-out view of the sample wherein the coalescence cascades are visible as intermittent dark ridge areas. Three bulges formed due to such events are encircled in panel (ii). These bulges grow due to repeated occurrences of the effect and coalescence with more droplets. Eventually, the resulting large drops [encircled in panel (iii)] are shed under gravity as indicated by yellow arrows. Drop 1 also moves under gravity initially but is eventually absorbed into the microgroove when it gets connected to a large drop pinned at the edge of the sample via a filled microgroove (panels vii and viii). Scale bar: $2 \mathrm{~mm}$. Substrate: aluminum. Coating: PTFE. Images are captured at $50 \mathrm{fps}$.

distributions are shown in the form of beeswarm plots. Each plot, shown in a different colors and centered about the corresponding microgroove width value on the $x$ axis, describes the relative number of swept droplets as a function of ordinate $D$ for that microgroove. We observe that the distributions are qualitatively similar across the four microgroove depths. The distributions are biased toward smaller $D$ values indicating that there are more numbers of smaller droplets swept by the coalescence cascades. This is due to the inherently much larger number of smaller droplets in the ridge area along the microgroove edge compared to that of larger droplets, which is qualitatively similar to the droplet distribution in planar surface condensation. ${ }^{35}$ The microgroove geometry, however, affects the largest droplets that can be swept by the coalescence cascade as evident from the distributions extending to larger $D$ values for larger depths.

A droplet is swept from the ridge by the advancing meniscus within the microgroove when the Laplace pressure for the coalescing droplet $P_{\mathrm{D}}$ is higher than that for the advancing condensate meniscus inside the microgroove, $P_{\mathrm{M}}$. This condition allows us to estimate an upper bound $D_{\text {crit }}$ for $D$ as $D<D_{\text {crit }}=[(1 / 2 W+1 / 4 H) \sin (\theta-\pi / 2)+1 / 4 H]^{-1}$ where $H$ is the microgroove depth and $\theta$ is the advancing contact angle of the meniscus inside the microgroove. $D_{\text {crit }}(H$, $W, \theta)$ signifies the maximum droplet size that can be swept by a coalescence cascade occurring in a partially filled hydrophobic microgroove of depth $H$, width $W$, and contact angle $\theta$. This limit is estimated based on the Laplace pressure difference between the advancing meniscus within the microgroove and the droplet on the ridge area at the microgroove edge that is about to coalesce with it (see Section S7 for details). As depicted in Figure 3A, this value bounds most of the droplet size distribution for the microgroove geometries tested. Further, this upper bound increases as the depth of the microgroove increases due to reduction in the relative Laplace pressure of the meniscus inside the microgroove with an increase in the depth of the microgroove. $D_{\text {crit }}$ is shown as a grey-colored zone in Figure $3 \mathrm{~A}$ due to the inherent uncertainty in estimating the angle $\theta$. Most of the swept droplets lie below this limit (yellow-colored zone of the figure). Panels i-iii in Figure 3B schematically illustrate the sweeping of droplets with $D<D_{\text {crit }}$ by the advancing meniscus in the microgroove. However, a few droplets with $D>D_{\text {crit }}$ are also swept by the coalescence sequence as shown by the data points in the orange zone above the $D_{\text {crit }}$ limit in Figure 3A. Recognizing that the $D_{\text {crit }}$ value above does not account for local variations in meniscus curvature, we speculate that these larger droplets are swept when the coalescence sequence proceeds in a completely filled part of the microgroove. In such a state, the condensate morphology in the microgroove results in a relatively lower $P_{M}$ as compared to partially filled regions of the microgroove (see Section S7 for details). Additionally, any bulges formed during the coalescence sequence or a prior presence of a large droplet along the microgroove can create a low-pressure sink that aids in the sweeping of the droplets with $D>D_{\text {crit }}$. Consider, for example, the sweeping of droplet 5 due to the creation of a bulge in Figure 3B (panels iii and iv).

2.4. Periodicity of the Coalescence Cascade. When the coalescence sequence concludes, the low-pressure sink provided by the single large bulge (Section S8) triggers a spontaneous withdrawal of the condensate from the microgroove as elucidated in Figure 4A,B. Subsequently, condensation restarts in the resulting dewetted length of the microgroove, and the cycle consisting of the coalescence sequence and spontaneous dewetting of the microgroove through condensate withdrawal is repeated all over again (Movie S3). Such bulges grow as they collect the condensate from the microgroove and coalesce with neighboring droplets outside the microgroove, eventually evolving into large drops (panels $\mathrm{i}$ and ii in Figure 4C), which are then shed from the 

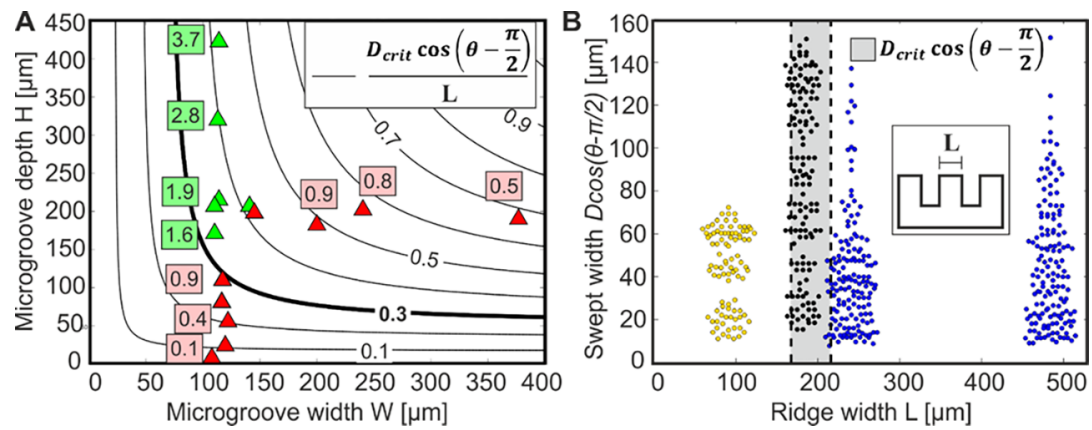

Figure 5. (A) Regime map of the liquid zipping-like coalescence sequence with varying aspect ratios $(H / W)$ and a fixed ridge width for hydrophobic microgrooves in aluminum. The numbers in green and red indicate aspect ratios for the various microgroove geometries. The green data points correspond to microgrooves for which the coalescence sequence is realized and red data points represent microgrooves where the coalescence sequence is absent. The contour lines correspond to the ratio $\left[D_{\text {crit }} \cos (\theta-\pi / 2)\right] / L$. Ridge width $L$ is defined in the inset of (B). Based on data points and contours, the microgrooves need to be designed such that $(H / W)>1.6$ and $\left[D_{\text {crit }} \cos (\theta-\pi / 2)\right] / L>0.3$. (B) Control of swept droplet size through reduction in microgroove ridge width $L$. Beeswarm distributions of swept droplet diameters are shown in terms of base diameter $D \cos (\theta-\pi / 2)$ for $\sim 100 \mu \mathrm{m}$ wide and $\sim 200 \mu \mathrm{m}$ deep microgrooves for ridge widths $L$ of $\sim 480$ and $\sim 240 \mu \mathrm{m}$ (in blue), $\sim 180 \mu \mathrm{m}$ (in black), and $\sim 90 \mu \mathrm{m}$ (in orange). $D_{\text {crit }} \cos (\theta-\pi / 2)$ for this microgroove geometry lies in the range of $\sim 164-216 \mu \mathrm{m}$ as indicated by the grey zone. For $L>D_{\text {crit }} \cos (\theta-\pi / 2)$, swept droplet distributions (in blue) are unaffected by $L$. However as $L$ is reduced, the swept droplet distribution first becomes more uniform [see distribution in black for $L \approx D_{\text {crit }} \cos (\theta-\pi / 2)$ ], and subsequently, the maximum droplet swept size is reduced when $L<D_{\text {crit }} \cos (\theta-\pi / 2)$ (see distribution in orange).

surface under gravity (Movie S4). Due to the multiple coalescences required to grow these drops to the eventual departure size, these drops are larger than the $D_{\text {crit }}$ limit for droplet sweeping from the ridge area for an individual coalescence cascade. In essence, a body force is required only once every few occurrences of the coalescence sequencecondensate withdrawal cycle to shed the large drops. This periodic cycle can sweep a large surface area over time compared to conventional dropwise condensation on planar hydrophobic samples (see Section S9). Although the zippinglike coalescence cascades cause wetting of the microgroove, it is unlike the wetting of microtextures observed in the case of droplets much larger in size than surface microfeatures and involving a change of the wetting state of the droplet from the Cassie-Baxter to the Wenzel state. ${ }^{36}$ In our work, the condensate droplets under consideration are of the same or smaller length scale than that of the microgrooves and always have the same wetting state as that on a planar hydrophobic surface. Additionally, unlike the irreversible change of the large-drop wetting state from Cassie-Baxter to Wenzel, the wetting of microgrooves described here is reversible as the morphology of the microgrooves causes its spontaneous dewetting due to the withdrawal of condensate as elucidated above.

2.5. Surface Design. To inspire rational design of surfaces for achieving this coalescence sequence, we investigated water condensation on hydrophobic microgrooves with aspect ratios (defined as $H / W$ ) spanning from 0.1 to nearly 4 with the ridge width kept constant. We observed that long coalescence sequences are consistently exhibited when $(H / W)$ is larger than $\sim 1.6$ and $D_{\text {crit }}$ is large enough such that $\left[D_{\text {crit }} \cos (\theta-\pi /\right.$ $2)] / L>\sim 0.3$ as illustrated in the regime map shown in Figure 5A. Here, $D_{\text {crit }} \cos (\theta-\pi / 2)$ represents the droplet base diameter corresponding to $D_{\text {crit }}$ and $L$ is the ridge width. At smaller aspect ratios or smaller $D_{\text {crit }} \cos (\theta-\pi / 2)$ values, the microgrooves cannot sustain a progressive meniscus movement necessary for the emergence of the cascading coalescence sequence, and the overall condensation process approaches the case of planar hydrophobic surfaces wherein such rapid droplet sweeping is absent (Section S10). Additionally, we observed that the maximum size of droplets swept by the liquid zipping effect could be controlled by reducing the ridge width between the microgrooves as shown in Figure $5 \mathrm{~B}$. As the ridge width is reduced below the $D_{\text {crit }} \cos (\theta-\pi / 2)$ limit, the maximum size that the droplets can grow before they are swept reduces, ${ }^{20}$ resulting in direct control over the swept droplet distribution.

\section{CONCLUSIONS}

We have elucidated a mechanism for passive removal of condensed droplets involving self-generated coalescence cascades of droplets along hydrophobic microgrooves. Such coalescence cascades offer an unexplored pathway for enhanced dropwise condensation without requiring traditional surface modification routes of passive condensate shedding adopted to date, involving either the fabrication of superhydrophobic textures, lubricant-impregnated textures, or surfaces with wettability gradients. We have quantified the droplet sweeping size as a function of microgroove geometry and have also proposed simple design rules for consistent realization of these coalescence cascades. Lastly, we have demonstrated control over the droplet removal size through modification of geometrical parameters of the texture. This control over the swept droplet size, and the fact that the periodic coalescence sequence and condensate removal cycle can sweep large areas of surfaces without the need for conventional droplet shedding modes (see Section S10) signals toward a potential to achieve significant enhancement in efficiency of industrial processes involving condensation. Additionally, we have noticed that droplets even as small as $\sim 10 \mu \mathrm{m}$ can sustain this sequential coalescence sequence (see Movie S5), indicating that it may be possible to realize this mechanism at even smaller scales by further downscaling the overall microgroove dimensions with suitable aspect ratios. This would be advantageous in miniaturized phase-change applications such as micro heat pipes ${ }^{37}$ and thermal diodes. ${ }^{21}$ Moreover, since this mechanism requires only standard hydrophobic (and not superhydrophobic) microgrooves, it accords significant flexibility regarding the choice of base material and coating for implementation in industrially relevant operating conditions. ${ }^{38}$ 


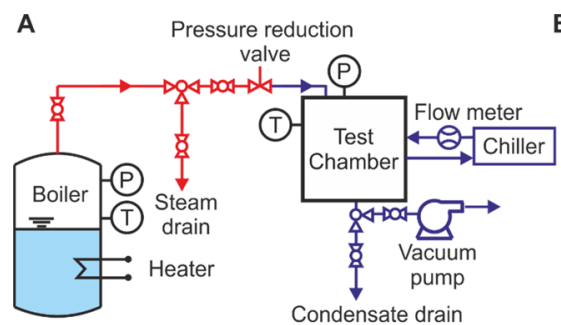

B

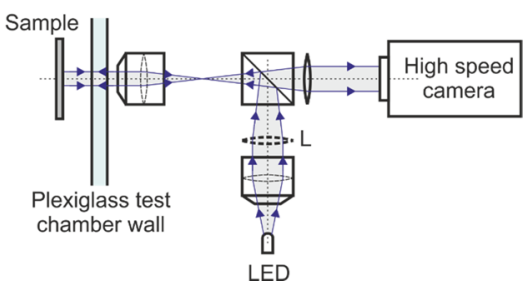

Figure 6. (A) Schematic of the experimental setup to induce condensation on the test sample. (B) Optical setup for high-speed, high-magnification observation of the domino effect. The lens $\mathrm{L}$, shown as dashed lines, was removed for imaging at high frame rates.

\section{EXPERIMENTAL SECTION}

4.1. Materials and Fabrication. Aluminum samples were cut from $1 \mathrm{~mm}$ thick sheets (99.5\% aluminum sheets, AW1085, Metal Service Menziken AG, Switzerland), and the microgrooves of various aspect ratios were milled into the sample. Subsequently, the samples were thoroughly cleaned by ultrasonication first in acetone and isopropanol (Sigma-Aldrich) and finally in DI water. The microgrooves were hydrophobized through a coating of either polytetrafluoroethylene (PTFE) or trichloro-1H,1H,2H,2H-perfluorodecylsilan (FDTS) (Sigma-Aldrich). For PTFE coating, the cleaned samples were first activated with oxygen plasma and then spray-coated with PTFE dispersed in dichloromethane (1 wt \%) (Sigma-Aldrich). The PTFE dispersion $(10 \mathrm{~mL})$ was sprayed over a sample area of approximately $30 \mathrm{~cm}^{2}$. The samples were sprayed using a VL doubleaction, internal mix, siphon-feed airbrush (Paasche, U.S.A.). The spray distance from the substrate was approximately $10 \mathrm{~cm}$, and the air pressure was set at $430 \mathrm{kPa}$. Subsequently, the samples were cured in an oven at $400{ }^{\circ} \mathrm{C}$ for $30 \mathrm{~min}$ to form a uniform hydrophobic coating on the sample. For a coating of FDTS, which forms a selfassembled monolayer by attaching with the surface hydroxyl groups, FDTS was dissolved in $n$-hexane (Sigma-Aldrich) to form a $1.43 \mathrm{mM}$ solution. The samples were kept immersed in this solution for 120 min and subsequently baked at $120{ }^{\circ} \mathrm{C}$ for $45 \mathrm{~min} .{ }^{17}$

Similar microgrooves were also fabricated in silicon by etching silicon wafers through a Bosch process using the PlasmaPro Estrelas 100 deep Si etcher (Oxford Instruments). Prior to that, a sacrificial layer was applied by spin-coating and patterned with a UV mask aligner Karl Suss MA6. It consisted of a positive photoresist (Microposit S1813). The unexposed parts were washed away using a solution containing $80 \mathrm{wt} \%$ water and $20 \mathrm{wt} \%$ Microposit 351 developer. The photoresist residues left under the etching process were subsequently removed by $\mathrm{O}_{2}$ plasma exposure at $600 \mathrm{~W}$ for 16 min (Q235 Microwave Plasma Etcher, Omni Technologies, U.S.A.). The etched samples were made hydrophobic by coating the sample with a thin layer of FDTS. The coating procedure was same as that for aluminum samples except that the immersion time was chosen as 2 min and the subsequent baking time was set as $10 \mathrm{~min}^{39}$

The PTFE-coated planar aluminum surface showed an advancing contact angle of $119.7^{\circ} \pm 8.2^{\circ}$ and a contact angle hysteresis of $31.1^{\circ}$ $\pm 4.8^{\circ}$. The FDTS-coated aluminum surface showed an advancing contact angle of $111.3^{\circ} \pm 8.0^{\circ}$ and a contact angle hysteresis of $37.8^{\circ}$ $\pm 8.1^{\circ}$, and the FDTS-coated silicon surface had an advancing contact angle of $119.8^{\circ} \pm 4^{\circ}$ and contact angle hysteresis of $43^{\circ} \pm 7.5^{\circ}$. The zipping-like coalescence cascades were observed on all three types of samples, which indicated that the occurrence of this effect is independent of the substrate material as long as the microgroove aspect ratio is correctly selected and all the surfaces of the microgroove are hydrophobic.

4.2. Experimental Setup. Figure 6A shows details of the custombuilt experimental setup. It consists of a boiler, a pressure-reducing valve, a Plexiglas test chamber, a chiller, and a vacuum pump. Deionized (DI) water is used as the working fluid. The boiler generates steam at $100{ }^{\circ} \mathrm{C}$ and 1 bar by heating the DI water with two $2 \mathrm{~kW}$ electrical heaters. The generated steam is supplied to the pressure-reducing valve through an insulated steam line (shown in red). The pressure-reducing valve (bellow valve, Swagelok) reduces the pressure of the steam, which is then supplied to the test chamber. The test chamber is evacuated using the vacuum pump prior to the supply of steam. This fluid circuit is designed to be an open loop such that the condensate is drained from the bottom of the test chamber. The sample is mounted in a vertical orientation with the microgrooves also aligned vertically, that is, parallel to gravity. During the experiment, the test chamber is maintained at a constant steam saturation pressure of $\sim 50 \mathrm{mbar}$, the chiller is set to a temperature of $10^{\circ} \mathrm{C}$, and the resulting sample temperature is $\sim 29^{\circ} \mathrm{C}$, indicating a surface subcooling of $4{ }^{\circ} \mathrm{C}$ (i.e., an oversaturation of $\sim 1.25$ ).

Figure $6 \mathrm{~B}$ shows a schematic of the optical microscope setup. A white LED is used as the light source that is collimated by using an objective. The collimated light is used to illuminate the sample using a beam splitter and through a long working distance objective (Olympus UPlanFl 4X/0.13). The signal from the sample is recorded by a high-speed camera (Photron Fastcam SA 1.1). The frame rate of the camera was adjusted according to the temporal information required from optical observations. For each frame rate setting, the maximum available image size setting was used. For observations of condensation on the entire sample, frame rates up to $250 \mathrm{fps}$ were used. On the other hand, to temporally resolve the high-speed capillary ripples on the meniscus pinned at the microgroove, a frame rate of 250,000 fps was used. All images were captured with a spatial resolution of $\sim 3.5$ microns/pixel. Refer to Section S2 for details of the test chamber and experimental protocol.

\section{ASSOCIATED CONTENT}

\section{S Supporting Information}

The Supporting Information is available free of charge on the ACS Publications website at DOI: 10.1021/acsami.9b07673.

Further details on materials, fabrication, and the experimental setup; experimental details on determination of perturbation amplitude; capillary ripple velocity; effect of the initial state of the microgroove on propagation speed of the cascading coalescence sequence; ESEM observations; long coalescence sequences and termination of the coalescence cascade; measurements of swept droplet distributions, analysis of meniscus morphology, and Laplace pressure differences during the coalescence sequence; mechanism of spontaneous condensate withdrawal from the microgroove; increase in the sweep area due to the coalescence sequence-condensate withdrawal cycle; role of the microgroove aspect ratio and ridge width in triggering the cascading coalescence sequence (PDF)

Cascading coalescence sequence along a hydrophobic microgroove that proceeds in a zipping-like motion to sweep droplets from the ridge area. Excess condensate is collected in two bulges that also eventually get absorbed into the microgroove due to the possible location of a larger bulge outside the field of view (AVI)

Initiation of the coalescence cascade captured through high-speed imaging at 250,000 fps. The movie shows 
that coalescence of a droplet outside the microgroove with the meniscus pinned at microgroove edges causes meniscus oscillations that trigger sequential coalescences of more droplets located along the microgroove edges (AVI)

The coalescence sequence ends with the microgroove completely filled with the condensate over the propagation length of the coalescence sequence, and excess condensate is collected in one large bulge. Subsequently, this bulge triggers spontaneous withdrawal of condensate from the microgroove, and condensation restarts in the cleared area of the microgroove (AVI)

Overall view of condensation on a $20 \mathrm{~mm} \times 20 \mathrm{~mm}$ microgrooved hydrophobic aluminum sample. Multiple occurrences of the cascading coalescence sequence and the shedding of resulting droplets are visible (AVI) Coalescence sequence triggered and sustained by small droplets on a microgroove filled with condensate (AVI) Comparison of propagation speed of the cascading coalescence sequence as a function of the state of the microgroove prior to initiation of condensation (AVI)

\section{AUTHOR INFORMATION}

\section{Corresponding Authors}

*E-mail: chander.sharma@iitrpr.ac.in. Phone: +91 1881 230108 (C.S.S.).

*E-mail: dpoulikakos@ethz.ch. Phone: +41 4463227 38. Fax: +41446321176 (D.P.).

\section{ORCID}

Chander Shekhar Sharma: 0000-0002-6193-6457

Dimos Poulikakos: 0000-0001-5733-6478

\section{Present Address}

${ }^{*}$ Department of Mechanical Engineering, Indian Institute of Technology Ropar, 140001 Rupnagar, India

\section{Author Contributions}

C.S.S. and D.P. conceived the research and provided scientific guidance in all of its aspects. C.S.S. designed the samples and experiments. A.M. developed the coating protocol and fabricated samples. H.E. designed the optical observation setup. C.W.E.L. and C.S.S. performed the experiments. C.S.S. and C.W.E.L. processed and analyzed the experimental results. C.S.S., C.W.E.L., and D.P. discussed the theoretical and data analyses, and C.S.S. wrote the first draft manuscript with subsequent inputs from all other coauthors leading to the final manuscript.

Notes

The authors declare no competing financial interest.

\section{ACKNOWLEDGMENTS}

C.S.S. acknowledges funding from the Commission for Technology and Innovation (CTI) under the Swiss Competence Centers for Energy Research (SCCER) program (grant no. KTI.2014.0148). A.M. acknowledges funding from the ERC advanced grant (grant no. 669908 INTICE) and SCCER (grant no. KTI.2014.0148). C.W.E.L acknowledges funding from the European Research Council (ERC) FET open grant (grant no. 801229 HARMoNIC). H.E. acknowledges funding from ETH Zurich. We thank Asel Maria Aguilar Sanchez, ETH Zurich, for her support of ESEM measurements and Jovo Vidic and Peter Feusi, ETH Zurich, for their assistance in construction of the condensation experimental setup. We also thank Willy Staubli, ETH Zurich, for machining the microgrooves in our samples.

\section{REFERENCES}

(1) International Energy Outlook 2016; U.S. Energy Information Administration: Washington, DC, 20585, 2016.

(2) The United Nations World Water Development Report 2015: Water for a Sustainable World; United Nations Educational, Scientific and Cultural Organization: Paris, 2015.

(3) Koomey, J. G. Growth in Data Center Electricity Use 2005 to 2010; Oakland, CA: Analytics Press, 2011.

(4) Kehlhofer, R.; Rukes, B.; Hannemann, F.; Stirnimann, F. Combined-Cycle Gas \& Steam Turbine Power Plants; 3rd ed.; PennWell, 2009.

(5) Alkhudhiri, A.; Darwish, N.; Hilal, N. Membrane Distillation: A Comprehensive Review. Desalination 2012, 287, 2-18.

(6) Kim, H.; Yang, S.; Rao, S. R.; Narayanan, S.; Kapustin, E. A.; Furukawa, H.; Umans, A. S.; Yaghi, O. M.; Wang, E. N. Water Harvesting from Air with Metal-Organic Frameworks Powered by Natural Sunlight. Science 2017, 356, 430-434.

(7) Davis, A.; Liakos, I.; Genovese, M. E.; Marini, L.; Salerno, M.; Bayer, I. S.; Athanassiou, A. Water Collection by Sticky Microislands on Superomniphobic Electrospun Surfaces. Adv. Mater. Interfaces 2016, 3, 1600606.

(8) Oh, J.; Birbarah, P.; Foulkes, T.; Yin, S. L.; Rentauskas, M.; Neely, J.; Pilawa-Podgurski, R. C. N.; Miljkovic, N. Jumping-Droplet Electronics Hot-Spot Cooling. Appl. Phys. Lett. 2017, 110, 123107.

(9) Rose, J. W. Dropwise Condensation Theory and Experiment: A Review. Proc. Inst. Mech. Eng., Part A 2002, 216, 115-128.

(10) de Gennes, P.-G.; Brochard-Wyart, F.; Quéré, D. Capillarity and Wetting Phenomena; Springer, 2004.

(11) Schellenberger, F.; Xie, J.; Encinas, N.; Hardy, A.; Klapper, M.; Papadopoulos, P.; Butt, H.-J.; Vollmer, D. Direct Observation of Drops on Slippery Lubricant-Infused Surfaces. Soft Matter 2015, 11, 7617-7626.

(12) Wong, T.-S.; Kang, S. H.; Tang, S. K. Y.; Smythe, E. J.; Hatton, B. D.; Grinthal, A.; Aizenberg, J. Bioinspired Self-Repairing Slippery Surfaces with Pressure-Stable Omniphobicity. Nature 2011, 477, 443-447.

(13) Dong, Z.; Schumann, M. F.; Hokkanen, M. J.; Chang, B.; Welle, A.; Zhou, Q.; Ras, R. H. A.; Xu, Z.; Wegener, M.; Levkin, P. A. Superoleophobic Slippery Lubricant-Infused Surfaces: Combining Two Extremes in the Same Surface. Adv. Mater. 2018, 30, 1803890.

(14) Li, H.; Aili, A.; Alhosani, M. H.; Ge, Q.; Zhang, T. Directional Passive Transport of Microdroplets in Oil-Infused Diverging Channels for Effective Condensate Removal. ACS Appl. Mater. Interfaces 2018, 10, 20910-20919.

(15) Quéré, D. Non-Sticking Drops. Rep. Prog. Phys. 2005, 68, 2495.

(16) Boreyko, J. B.; Chen, C.-H. Self-Propelled Dropwise Condensate on Superhydrophobic Surfaces. Phys. Rev. Lett. 2009, $103,184501$.

(17) Sharma, C. S.; Combe, J.; Giger, M.; Emmerich, T.; Poulikakos, D. Growth Rates and Spontaneous Navigation of Condensate Droplets Through Randomly Structured Textures. ACS Nano 2017, $11,1673-1682$.

(18) Wen, R.; Xu, S.; Zhao, D.; Lee, Y.-C.; Ma, X.; Yang, R. Hierarchical Superhydrophobic Surfaces with Micropatterned Nanowire Arrays for High-Efficiency Jumping Droplet Condensation. ACS Appl. Mater. Interfaces 2017, 9, 44911-44921.

(19) Daniel, S.; Chaudhury, M. K.; Chen, J. C. Fast Drop Movements Resulting from the Phase Change on a Gradient Surface. Science 2001, 291, 633-636.

(20) Ghosh, A.; Beaini, S.; Zhang, B. J.; Ganguly, R.; Megaridis, C. M. Enhancing Dropwise Condensation through Bioinspired Wettability Patterning. Langmuir 2014, 30, 13103-13115.

(21) Boreyko, J. B.; Zhao, Y.; Chen, C.-H. Planar Jumping-Drop Thermal Diodes. Appl. Phys. Lett. 2011, 99, 234105. 
(22) Park, K.-C.; Kim, P.; Grinthal, A.; He, N.; Fox, D.; Weaver, J. C.; Aizenberg, J. Condensation on Slippery Asymmetric Bumps. Nature 2016, 531, 78-82.

(23) Narhe, R. D.; Beysens, D. A. Nucleation and Growth on a Superhydrophobic Grooved Surface. Phys. Rev. Lett. 2004, 93, 076103.

(24) Brown, R. A.; Scriven, L. E. On the Multiple Equilibrium Shapes and Stability of an Interface Pinned on a Slot. J. Colloid Interface Sci. 1980, 78, 528-542.

(25) Kapur, N.; Gaskell, P. H. Morphology and Dynamics of Droplet Coalescence on a Surface. Phys. Rev. E 2007, 75, 56315.

(26) Landau, L. D.; Lifshitz, E. M. Fluid Mechanics; McGraw-Hill, Ed.; Course of Theoretical Physics; Pergamon Press, 1987; Vol. 6.

(27) Kenyon, K. E. Capillary Waves Understood by an Elementary Method. J. Oceanogr. 1998, 54, 343-346.

(28) Noblin, X.; Buguin, A.; Brochard-Wyart, F. Vibrated Sessile Drops: Transition between Pinned and Mobile Contact Line Oscillations. Eur. Phys. J. E: Soft Matter Biol. Phys. 2004, 14, 395-404.

(29) Sharp, J. S. Resonant Properties of Sessile Droplets; Contact Angle Dependence of the Resonant Frequency and Width in Glycerol/Water Mixtures. Soft Matter 2012, 8, 399-407.

(30) Aarts, D. G.; Lekkerkerker, H. N.; Guo, H.; Wegdam, G. H.; Bonn, D. Hydrodynamics of Droplet Coalescence. Phys. Rev. Lett. 2005, 95, 164503.

(31) Wu, M.; Cubaud, T.; Ho, C.-M. Scaling Law in Liquid Drop Coalescence Driven by Surface Tension. Phys. Fluids 2004, 16, L51L54.

(32) Andrieu, C.; Beysens, D. A.; Nikolayev, V. S.; Pomeau, Y. Coalescence of Sessile Drops. J. Fluid Mech. 2002, 453, 427-438.

(33) Narhe, R.; Beysens, D.; Nikolayev, V. S. Contact Line Dynamics in Drop Coalescence and Spreading. Langmuir 2004, 20, $1213-1221$.

(34) Bonn, D.; Eggers, J.; Indekeu, J.; Meunier, J.; Rolley, E. Wetting and Spreading. Rev. Mod. Phys. 2009, 81, 739-805.

(35) Le Fevre, E. J.; Rose, J. W. A Theory of Heat Transfer by Dropwise Condensation. In 3rd International Heat Transfer Conference; Begell House Inc.: Chicago, 1966; Vol. 2, p. 362.

(36) Sbragaglia, M.; Peters, A. M.; Pirat, C.; Borkent, B. M.; Lammertink, R. G. H.; Wessling, M.; Lohse, D. Spontaneous Breakdown of Superhydrophobicity. Phys. Rev. Lett. 2007, 99, 156001. (37) Qu, J.; Wu, H.; Cheng, P.; Wang, Q.; Sun, Q. Recent Advances in MEMS-Based Micro Heat Pipes. Int. J. Heat Mass Transfer 2017, 110, 294-313.

(38) Sharma, C. S.; Stamatopoulos, C.; Suter, R.; von Rohr, P. R.; Poulikakos, D. Rationally 3D-Textured Copper Surfaces for Laplace Pressure Imbalance-Induced Enhancement in Dropwise Condensation. ACS Appl. Mater. Interfaces 2018, 10, 29127-29135.

(39) Maitra, T.; Tiwari, M. K.; Antonini, C.; Schoch, P.; Jung, S.; Eberle, P.; Poulikakos, D. On the Nanoengineering of Superhydrophobic and Impalement Resistant Surface Textures below the Freezing Temperature. Nano Lett. 2014, 14, 172-182. 